\title{
Review
}

\section{Research advances in reactivation of hepatitis virus after chemotherapy for non-Hodgkin's lymphoma-combined hepatitis B virus infection}

\author{
Shujun $\mathrm{Ma}$ \\ Department of Medical Oncology, Shandong University Affiliated Shandong Provincial Hospital, Jinan China
}

\author{
Keywords \\ Lymphoma, nonHodgkin's; hepatitis, hepatitis \\ $\mathrm{B}$, chronic; chemotherapy; rituximab

\section{Correspondence} \\ Shujun Ma, \\ E-mail: sjmajn@163.com \\ DOI: 10.1515/ii-2017-0111
}

\begin{abstract}
Infection rate of hepatitis B virus (HBV) in our country remains high. Many patients showed combined HBV infection; the most common blood system disease is non-Hodgkin' s lymphoma (NHL)-combined HBV infection. Drugs used in treating lymphoma may induce different degrees of HBV reactivation. Such condition may lead to hepatic failure or death. Currently, scholars pay increasing attention to reactivation of HBV by rituximab and/or chemotherapy for NHL-combined HBV patients. This study summarizes research advances in this topic, with a view of providing background information for further research.
\end{abstract}

Hepatitis B virus (HBV) is a prevalent organism in our country. Many patients may feature combined HBV infection. Among HBV-infected patients, those with nonHodgkin's lymphoma (NHL)-combined HBV must be acknowledged. With continuous development of treatment drugs for lymphoma, most scholars focus on reactivation of $\mathrm{HBV}$ in NHL-combined $\mathrm{HBV}$ patients after receiving Mabthera (rituximab) and/or chemotherapy. In this study, we summarize research advances in relevant predisposing factors, mechanism of occurrence, and treatment and prevention of $\mathrm{HBV}$ infection.

\section{Status of patients with NHL- combined HBV infection}

HBV infection is distributed worldwide, mostly in Asia, Africa, Latin America, and other developing countries. In China, HBV infection is also prevalent. According to results of our epidemiological survey, HBV surface antigen-positive rate in Chinese population approximates $9.09 \%^{[1]}$.

HBV and lymphoid tissues are ecotropic. As lymphoma patients often feature low-level immune functions, they are more prone to $\mathrm{HBV}$ infection than the general population or patients with other tumors.

Currently, three hypotheses are mainly provided regarding the correlation between $\mathrm{HBV}$ infection and $\mathrm{NHL}^{[2]}$ : (1) direct immunosuppressive effects of lymphoma may lead to increased risk of $\mathrm{HBV}$ infection and reactivation; (2) HBV carriers may activate lymphoma pathogenicity gene; and (3) another unknown virus may be present, and its propagation mode, which is similar to that of $\mathrm{HBV}$, may lead to activation of lymphoma pathogenicity gene.

Epidemiological statistics showed that HBV surface antigen-positive rate of NHL patients reaches 24\%-77\% ${ }^{[3]}$. Case-control study of Kim et al. ${ }^{[4]}$ showed that among 222 newly diagnosed NHL patients, HBV surface antigenpositive rate totaled $12.6 \%$, whereas rates in concurrently controlled 439 cases of nonblood system tumors and 444 cases of nontumor patients reached $7.3 \%$ and $4.7 \%$, respectively. Therefore, NHL and HBV present statistically significant correlation. In recent years, epidemiological statistics showed that incidence of NHL increases at a rate of $4 \%$ each year ${ }^{[2]}$. Thus, increasing attention centered on populations with NHL-combined HBV infection.

\section{Status of NHL chemotherapy}

Cyclophosphamide, doxorubicin, vincristine, and prednisone (CHOP) are regarded the standard regimens for $\mathrm{NHL}$ patients, with overall response rate of $80 \%-90 \%$, complete 
remission rate of $45 \%-55 \%$, and five-year survival rate of $30 \%$ $-40 \%{ }^{[5]}$. Invention of rituximab (product name: Mabthera) provided a new option for NHL treatment.

As the first monoclonal antibody applied in NHL clinical treatment, rituximab displays advantages, including high monotherapy efficiency, good safety, no dose-limiting toxicity, and bone marrow hematopoietic stem cell toxicity. Rituximab can also be used in combination with chemotherapeutic drugs to enhance sensibility of tumor cells to chemotherapeutic drugs.

Recently, multiple large-scale clinical tests were conducted to assess therapeutic effects of rituximab and CHOP (R-CHOP) as the first-line regimen for NHL. MinT test ${ }^{[6]}$ was used to assess therapeutic effects of rituximab combined with CHOP-like chemotherapy or single CHOPlike chemotherapy among 824 young patients of diffuse large B-cell lymphoma with improved prognosis. Results showed that within a median follow-up period of 34 months, three-year event-free survival rate (EFS) (79\% and 59\%, respectively, in rituximab combined with $\mathrm{CHOP}$-like chemotherapy group and chemotherapy group, $P<0.0001)$ and overall survival rate (OS) (93\% and $84 \%$, respectively, in rituximab combined with $\mathrm{CHOP}$-like chemotherapy group and chemotherapy group, $P=0.0001$ ) of patients who received rituximab with chemotherapy were all superior to those of single-chemotherapy group. The French Study Group of the Adult Lymphoma test ${ }^{[7]}$ conducted a sevenyear follow-up for untreated elderly NHL patients treated with R-CHOP and CHOP regimens. Results showed that EFS rate ( $42 \%$ and $25 \%$ in $\mathrm{R}-\mathrm{CHOP}$ group and $\mathrm{CHOP}$ group, respectively; $P=0.000$ 02), progression-free survival rate (52\% and 29\% in R-CHOP and CHOP groups, respectively; $P<0.0001)$, disease-free survival rate $(66 \%$ and $42 \%$, in R-CHOP and CHOP groups, respectively; $P<0.0001)$, and OS rates (53\% and $36 \%$ in $\mathrm{R}-\mathrm{CHOP}$ and $\mathrm{CHOP}$ groups, respectively; $\mathrm{P}=0.0004$ ) of $\mathrm{R}-\mathrm{CHOP}$ group were superior to those of the CHOP group. These large-scale clinical tests showed that R-CHOP exhibits remarkable advantages than $\mathrm{CHOP}$. As a result, $\mathrm{R}-\mathrm{CHOP}$ regimen became widely used in clinical practice.

\section{Reactivation of HBV}

Reactivation of $\mathrm{HBV}$ is based on detection of valence of $\mathrm{HBV}$ surface antigen and HBV surface antibody. Two independent clinical hypotheses during immunosuppressive therapy are proposed: (1) increased valence of HBV surface antigen in HBV surface antigen-positive patients; and (2) HBV surface antigen-negative and/or HBV surface antibody-positive patients feature surface antibody loss accompanied by reproduction of $\mathrm{HBV}$ surface antigen (serotype conversion). With progression of $\mathrm{HBV}-\mathrm{DNA}$ quantitative analysis, $\mathrm{HBV}$ reactivation can be tracked through correlation between HBV-DNA levels and hepatitis and chemotherapy time.

Currently, $\mathrm{HBV}$ reactivation is defined as $\mathrm{HBV}$ replication during cytotoxic or immunosuppressive therapy or closely thereafter in chronic HBV patients combined tumor [HBV -DNA is elevated over 10 times, or its absolute value $>10^{9}$ copies/mL, with low detection limit of branched DNA method as $7.0 \times 10^{5}$ copies $/ \mathrm{mL}$, whereas polymerase chain reaction (PCR) detects 300 copies $/ \mathrm{mL}$ ], excluding other infections ${ }^{[8]}$. After chemotherapy, $\mathrm{HBV}$ reactivation may cause continuous necrosis of liver cells and progressive decrease in liver functions, eventually leading to hepatic damage with varied severity. In less severe cases, HBV reactivation may induce hepatitis symptoms, such as elevated liver enzymes or jaundice; in severe cases, this condition may lead to fulminant hepatic failure or death ${ }^{[8]}$.

Reactivation of HBV generally occurs in HBV surface antigen-positive patients. $\mathrm{HBV}$ reactivation may also occur in $\mathrm{HBV}$ surface antigen-negative patients, HBVe antibody and $\mathrm{HBV}$ surface antigen-negative patients, and $\mathrm{HBV}$ core antibody-positive patients.

In general, two routes exist for $\mathrm{HBV}$ reactivation ${ }^{[9,10]}$ : (1) cytotoxic drugs and immunosuppressive agents inhibit immunologic mechanism that controls $\mathrm{HBV}$ replication, thereby leading to massive replication of HBV; (2) after controlling patients' condition, immune function gradually recovers, thereby causing damage to immune-mediated infected liver cells. With two different routes, HBV reactivation can be divided into two stages ${ }^{[9,11]}$. The first stage occurs during intensive chemotherapy with cytotoxic drugs and immunosuppressive agents and is characterized by replication and increase in $\mathrm{HBV}$ and mainly manifested as increase in serum $\mathrm{HBV}-\mathrm{DNA}$, HBVe antigen and $\mathrm{HBV}$ -DNA polymerase, and HBV-infected normal liver cells; the second stage relates to recovery of immune function after discontinuation of medication, clinically manifested as symptoms of hepatitis-like elevated liver enzyme and jaundice, and may lead to hepatic failure or death in severe cases $^{[12]}$. 


\section{Reactivation of HBV after Mabthera and/or chemotherapy of NHL patients}

As mentioned, among NHL patients, several HBV-infected patients may present $\mathrm{HBV}$ reactivation after receiving chemotherapy. In surface antigen-positive patients with lymphoma, incidence of $\mathrm{HBV}$ reactivation after chemotherapy reaches $20 \%-55 \%$; this value is two to three times higher than that of other tumors ${ }^{[13]}$. Therefore, HBV reactivation is currently considered as one of the possible complications after chemotherapy of tumor patients, especially of NHL patients with chronic HBV infection. Factors that possibly induce HBV reactivation are as follows.

\section{Virological factors}

Currently, speculations indicate that presence of $\mathrm{HBVe}$ antigen is related to $\mathrm{HBV}$ reactivation. However, studies also showed that $\mathrm{HBVe}$ antigen-negative patients may also face increasing risk of reactivation because of variations in $\mathrm{HBV}$ pre- $\mathrm{C}$ zone and basic core promoter. Early studies indicated that viral load before chemotherapy is irrelevant to HBV reactivation post-chemotherapy. With application of methods including PCR, new insights into this conclusion became available. Study by Zhong et al. ${ }^{[14]}$ showed that high-serum HBV-DNA load ( $>3 \times 10^{5}$ copies $/ \mathrm{mL}$ ) before chemotherapy increases possibility of $\mathrm{HBV}$ reactivation. Currently, some conclusions were formed concerning covalently closed circular DNA of HBV (HBV cccDNA), i.e., HBV cccDNA is an existing form of $\mathrm{HBV}$ in humans. As an original copy template of $\mathrm{HBV}, \mathrm{HBV}$ cccDNA plays a significant role in $\mathrm{HBV}$ replication and establishment of infection status. As long as HBV cccDNA exists in liver cells, HBV replication continues. Currently, most used antiviral drugs can only inhibit HBV cccDNA. With its long half-life (33-70 days $\left.{ }^{[15]}\right), \mathrm{HBV}$ is not easily eliminated. Thus, once medication is discontinued or comes the outer simulations, disease relapse occurs ${ }^{[16]}$. Hui et al. ${ }^{[17]}$ raised that high levels of cccDNA in the liver may be used to predict $\mathrm{HBV}$ reactivation in $\mathrm{HBV}$ surface antigen-positive patients after chemotherapy.

\section{Antineoplastic drugs}

Two types of available antineoplastic drugs may induce HBV reactivation: traditional cytotoxic drugs and biological agents related to anti-B-cell and anti-T-cell monoclonal antibody treatment.

Among traditional cytotoxic drugs, corticosteroids and anthracycline drugs present the closest correlation with HBV reactivation ${ }^{[18]}$. Corticosteroids and glucocorticoids easily lead to $\mathrm{HBV}$ reactivation, possibly because they can directly promote $\mathrm{HBV}$ replication by acting on corticosteroid response element of $\mathrm{HBV}$ genome. These drugs can inhibit normal lymphocyte function and immune function by inducing DNA degradation, inhibiting substance metabolism, and inducing apoptosis in lymphocytes, thereby leading to $\mathrm{HBV}$ reactivation. Anthracycline drugs stimulate HepG2.2.15 cells to secrete HBV-DNA in a doserelated manner. This phenomenon increases incidence of HBV reactivation when patients diagnosed with HBV use anthracycline drugs. NHL patients most commonly use corticosteroids and anthracycline drugs as chemotherapeutic drugs. These compounds are also used in combination in most cases. Thus, during chemotherapy, NHL patients exhibit much higher incidence of $\mathrm{HBV}$ reactivation than those with other diseases.

As for anti-B-cell and anti-T-cell monoclonal antibodies, anti-CD20 monoclonal antibody (rituximab) is the most commonly used drug with definite curative effects; it also serves as the first-line drug treatment for CD20-positive diffuse large B-cell lymphoma. CD20 is expressed on surfaces of all pre-B cells and mature B cells. Rituximab binds to CD20 antigen on B lymphocytes through complementdependent cytotoxicity and antibody-dependent cellmediated cytotoxicity. Rituximab inhibits proliferation of B lymphocytes and induces their dissolution and apoptosis. These actions may directly lead to a decrease in the number of plasmocytes differentiating from B lymphocytes, followed by a decrease in generation of antibodies. This phenomenon helps create favorable conditions for $\mathrm{HBV}$ reactivation after chemotherapy. Dai et al. ${ }^{[19]}$ provided six courses of R-CHOP chemotherapy to four patients with HBV surface antigen-positive diffuse large B-cell lymphoma. These researchers also used lamivudine for antiviral therapy during chemotherapy, conducted follow-up visits for 14 months, and discontinued lamivudine treatment after six-month chemotherapy. Results showed that within one month after medication was stopped, four patients showed different degrees of HBV reactivation (abnormal increase in glutamic -pyruvic transaminase and HBV-DNA), indicating that rituximab combined with chemotherapy can induce $\mathrm{HBV}$ reactivation. Tsutsumi et al. ${ }^{[20]}$ analyzed five diffuse large B-cell lymphoma patients receiving rituximab treatment and observed that all patients presented different degrees of 
decrease in valence of $\mathrm{HBV}$ surface antibody and increased levels of glutamic-pyruvic transaminase. One HBV surface antigen-positive patient and who did not receive antiviral therapy manifested liver failure and died because of serious liver function damage. Tsutsumi et al. ${ }^{[20]}$ analyzed research results in recent years and discovered that not only the HBV surface antigen-positive or HBVe antigen-positive patients with replication of HBV-DNA showed different degrees of $\mathrm{HBV}$ reactivation after receiving rituximab treatment but also those who were HBV surface antibody-positive, HBVe antigen-positive, and $\mathrm{HBV}$ c antigen-positive and received HBV reactivation treatment. Law et al. ${ }^{[21]}$ obtained results that agreed with those of Tsutsumi et al. ${ }^{[20]}$. Their study also showed that both single use of rituximab and its combined use with cytotoxic drugs induce reactivation of existing $\mathrm{HBV}$. Although incidences between these two treatments were not statistically significant (the former results in $40 \%$ chance of $\mathrm{HBV}$ reactivation, and the latter approximates $38 \%$ ), degree of severity of $\mathrm{HBV}$ reactivation induced by single use of rituximab was slightly lower than that induced by its combined use with cytotoxic drugs.

\section{Prevention and treatment of HBV reactivation}

Previously, interferon was used for prevention and treatment of $\mathrm{HBV}$ reactivation. However, this treatment features weak direct antiviral effects and notable adverse reactions and poor compliance of patients. Interferon may enhance immunomodulatory damage in liver cells and lead to fatal hepatitis; thus, it is not widely used ${ }^{[18]}$. In recent years, scholars at home and abroad reported that the nucleoside analog lamivudine exhibited good therapeutic effect on $\mathrm{HBV}$ reactivation, and prophylactic use of drug before chemotherapy displayed better effects than using the drug after increase in HBV-DNA ${ }^{[22,23]}$. Hsu et al. ${ }^{[24]}$ randomly divided $52 \mathrm{NHL} / \mathrm{HBV}$ carriers without treatment into two groups (prevention and treatment groups). Both groups received six courses of CHOP chemotherapy. The prevention group used lamivudine in combination with chemotherapy for prevention (oral treatment of $0.1 \mathrm{~g}$ lamivudine, once a day from the first day of chemotherapy to two months after the end). The treatment group received lamivudine only after presenting $\mathrm{HBV}$ reactivation (with administration route and dose similar to those of prevention group and continuous administration until recovery from hepatitis). Patients were followed up for 12 months, and results showed that incidences of reactivation of $\mathrm{HBV}$ in prevention and treatment groups reached $11.5 \%$ and $56.0 \%(\mathrm{P}=0.0001)$, respectively. In the prevention group, time from receiving chemotherapy to emergence of $\mathrm{HBV}$ was longer than that of treatment group $(P=0.018)$. He et al. ${ }^{[25]}$ included $29 \mathrm{NHL} / \mathrm{HBV}$ carriers in their study (28 received $\mathrm{R}-\mathrm{CHOP}$ chemotherapy, and one received rituximab, fludarabine, mitoxantrone, and dexamethasone chemotherapy). All patients received lamivudine a week before the start of chemotherapy until two months after the end of treatment (oral administration of $0.1 \mathrm{~g}$ lamivudine, once a day). Patients were followed up for 16 months; four (13.8\%) showed liver damage, but none showed increase in replication of HBV-DNA. Loomba et al. ${ }^{[26]}$ summarized data and results of relevant tests in recent years and pointed out that lamivudine can effectively prevent HBV reactivation. After chemotherapy, lamivudine also significantly reduced incidence of $\mathrm{HBV}$ reactivation by $79 \%$ in patients with $\mathrm{HBV}$ surface antigen-positive tumor .

Currently, for HBV surface antigen-positive patients, the recognized regimen is administering lamivudine $(0.1 \mathrm{~g}$ oral administration, once a day) as antiviral therapy a week before chemotherapy until four to six weeks after the end of treatment or the time determined as per patients' conditions $[27,28]$. The lately published The Guideline of Prevention and Treatment for Chronic Hepatitis $B{ }^{[29]}$ corrects the duration of antiviral therapy. The guideline recommends that course of antiviral therapy should start at the beginning of immunosuppressive or chemotherapeutic therapy to at least 12 weeks after receiving such treatment. For HBV surface antigen-negative and other antibody-positive patients, studies should still clarify whether preventive antiviral therapy should be provided before using immunosuppressive agents or chemotherapy. When HBV reactivation occurs after chemotherapy, chemotherapy should be stopped immediately, and antiviral drugs, such as lamivudine, should be used consecutively for one-year treatment. During this period, liver function and viral indicators should be reexamined regularly.

Although lamivudine exhibits advantages on prevention and treatment of $\mathrm{HBV}$ reactivation, it features some limitations, such as drug resistance and virus variations at prolonged administration time and occurrence of withdrawal hepatitis after discontinuation of medication. This phenomenon drives scholars to develop safe and 
effective drugs. To date, in comparison with lamivudine, lower incidences of drug resistance are observed when using adefovir and entecavir in treatment of chronic HBV infection. However, insufficient treatment data can describe effectivity of these drugs in preventing reactivation of HBV. Thus, in this regard, long-term follow-up studies are required for further assessment.

\section{Conclusion}

In NHL patients with combined HBV infection, HBV reactivation is one of the potential complications after rituximab and/or chemotherapy. Therefore, to reduce negative effects of $\mathrm{HBV}$ reactivation and to treat primary blood system diseases, patients must be treated with antiviral drugs during chemotherapy.

\section{Declarations}

\section{Acknowledgements}

No.

\section{Competing interests}

The author declares that he has no competing interest.

\section{Authors' contributions}

SJ Ma made the literature analysis and wrote, discussed and revised the manuscript of this review.

\section{References}

1 Chinese Society of Hepatology of Chinese Medical Association, Chinese Society of Infectious Diseases of Chinese Medical Association. The Guideline of Prevention and Treatment for Chronic Hepatitis B. Chinese Journal of Internal Medicine, 2006, 45(2):162-170.

2 Chen Y, Xie Q, Shen Z. Reactivation of HBV Related to Treatment of Malignant Lymphoma. Journal of Internal Medicine Concepts \& Practice, 2007, 2(1):8-12.

3 Zheng R, Qin F, Zhai Y, et al. HBV Infection and Chemotherapy of NonHodgkin's Lymphoma. The Practical Journal of Cancer, 2001, 16(2):181.

4 Kin JH, Bang YJ, Parb J, et al. Hepatitis B virus infection and B-cell nonHodgkin' s lymphoma in a hepatitis B endemic area: a case-control study. Jpn J Cancer Res, 2002, 93(5): 471-477.

5 Guan Z, Wang S. Research Status of Chemotherapy for B Cell Lymphoma. Chinese Journal of Oncology, 2005, 27(12):760-761.

6 Pfreundschuh M, Trumper L, Osterborg A, et al. CHOP-like chemotherapy plus rituximab versus CHOP-like chemotherapy alone in young patients with good-prognosis diffuse large B-cell lymphoma: a randomised controlled trial by the MabThera International Trial (MinT) Group. Lancet Oncol, 2006, 7(5):379-39.

7 Coiffier B, Feugier P, Mounier N, et al. Long-term results of the GELA study comparing RCHOP and CHOP chemotherapy in older patients with diffuse large B-cell lymphoma show good survival in poor-risk patients. Proc ASCO, 2007, 25(18S):443a.

8 YEO W, JOHNSON P J. Diagnosis, prevention and management of hepatitis B virus reactivation during anticancer therapy .Hepatology,2006, 43(2):209-220.

9 Hui CK, Cheung WW, Au WY, et al. Hepatitis B reactivation after withdrawal of pre-emptive lamivudine in patients with haematological malignancy on completion of cytotoxic chemotherapy. Gut, 2005, 54(11):1597-1603.

10 Cheng AL, Hsiung CA, Su IJ, et al. A prospective randomized study comparing steroid-containing versus steroid-free chemotherapy in the treatment of hepatitis B virus carriers with malignant lymphoma. Hepatology, 2003, 37(6):1320-1328.

11 Liang X, Wan M, Chen J, et al. Therapy and Prevention Effect of Lamivudine on HBV Reactivation in HBsAg Carrier with Immune Default. Journal of Chinese Modern Medicine, 2005, 2(7):594-596.

12 Rossi G. Prophylaxis with lamivudine of hepatitis B virus reactivation in chronic HbsAg carriers with hemoto-oncological neoplasias treated with chemotherapy. Leuk Lymphoma, 2003, 44(5):759-766.

13 Yeo W, Chan PK, Zhong S, et al. Frequency of hepatitis B virus reactivation in cancer patients undergoing cytotoxic chemotherapy: a prospective study of 626 patients with identification of risk factors. J Med Virol, 2000, 62(3):209-307.

14 Zhong S, Yeo W, Schroder C, et al. High hepatitis B virus (HBV) DNA viral load is an important risk factor for HBV reactivation in breast cancer patients undergoing cytotoxic chemotherapy. J Viral Hepat, 2004, 11(1):55-59.

15 Addison WR, Walters KA, Wong WW, et al. Half-life of the duck hepatitis $B$ virus convalenly closed circular DNA pool in vivo following inhibition of viral replication . J Virol, 2002, 76(12):6356-6363.

16 Kock J, Baumert TF, Delaney WE, et al. Inhibitory effect of adfovir and lamivudine on the initiation of hepatitis B virus infection in primary tupaia hepatocytes. Hepatology, 2003, 38(6):1410-1418.

17 Hui CK, Bowden S, Jackson K, et al. Clinical significance of intrahepatic hepatitis B virus covalently closed circular DNA in chronic hepatitis B patients who received cytotoxic chemotherapy. Blood, 2005, 105(6):2616-2617.

18 Gui H, Xie Q. Diagnosis, Management and Prevention of hepatitis B virus reactivation during antitumor therapy. Chinese Journal of Hepatology, 2007, 15(1):78-80.

19 Dai MS, Chao TY, Kao WY, et al. Delayed hepatitis B virus reactivation after cessation of preemptive lamivudine in lymphoma patients treated 
with rituximab plus CHOP. Ann Hematol, 2004, 83(12):769-774.

20 Tsutsumi Y, Kanamori H, Mori A, et al. Reactivation of hepatitis B virus with rituximab . Expert Opin Drug Saf, 2005, 4(3):599-608.

21 Law JK, Ho JK, Hoskins PJ, et al. Fatal reactivation of hepatitis B postchemotherapy for lymphoma in a hepatitis B surface antigen- negative, hepatitis B core antibody--positive patient: potential implications for future prophylaxis recommendations. Leuk Lymphoma, 2005, 46(7):1085-1089. cancer patients carrying hepatitis B virus undergoing cytotoxic chemotherapy: a longitudinal study . Support Care Cancer, 2004, 12(3):191-196.

23 Liang X, Wan M, Chen J, et al. Therapy and Prevention Effect of Lamivudine on HBV Reactivation in HBsAg Carrier with Immune Default. Chinese Journal of Hepatology, 2005, 13(6):382-383.

24 Hsu C, Hsiung CA, Su IJ, et al. A revisit of prophylactic lamivudine for chemotherapy-associated hepatitis B reactivation in non-Hodgkin' s lymphoma: a randomized trial. Hepatology, 2008, 47(3):844-853.

$25 \mathrm{He}$ Y, Li Y, Wang FH, et al. The effectiveness of lamivudine in preventing hepatitis B viral reactivation in rituximab-containing regimen for lymphoma.Ann Hematol, 2008, 87 (6):481-485.

26 Loomba R, Rowley A, Wesley R, et al. Systematic review: the effect of preventive lamivudine on hepatitis $\mathrm{B}$ reactivation during chemotherapy. Ann Intern Med, 2008, 148 (7):519-528.

27 Lok AS, Mcmahon BJ. Chronic hepatitis B. Hepatology, 2007 , 45(2):507-539.

28 Sherman M, Sharran S, Burak K, et al. Management of chronic hepatitis B: Consensus guidelines. Can J Gastroenterol, 2007, 12(Suppl C):5C24C.

29 Jin Q, Niu J. Brief Introduction and Interpretation of 2008 APASL Guideline for HBV Management. Chinese Journal of Clinical Hepatology, 2008, 24(3):166-168. 